\title{
Calcipotriene-induced improvement in psoriasis is associated with reduced interleukin-8 and increased interleukin-10 levels within lesions
}

\author{
S.KANG, S.YI, C.E.M.GRIFFITHS, ${ }^{*}$ L.FANCHER, T.A.HAMILTON AND J.H.CHOI $\dagger$ \\ Dermatopharmacology Unit, Department of Dermatology, University of Michigan Medical Center, Ann Arbor, MI 48109-0314, \\ U.S.A. \\ *Section of Dermatology, University of Manchester School of Medicine, Manchester, U.K. \\ $\dagger$ Department of Dermatology, Asan Medical Center, Seoul, Korea \\ Accepted for publication 14 August 1997
}

Summary Calcipotriene is a synthetic analogue of 1,25-dihydroxyvitamin $\mathrm{D}_{3}$ established to be effective topically in the treatment of psoriasis. We investigated the early cellular and immunological events induced by calcipotriene in psoriasis. Thirty patients with moderate plaque-type psoriasis were randomly assigned to receive twice daily applications of either calcipotriene ointment $0 \cdot 005 \%$ or matching vehicle for 6 weeks. Skin biopsies $(6 \mathrm{~mm})$ were performed from designated plaques at baseline and days 3 and 7. On these days and at weeks 2, 4 and 6, complete clinical evaluations were made in a double-blind fashion. Consistent with previous studies, significant clinical improvement $(P<0 \cdot 05)$ in psoriasis was observed in patients receiving calcipotriene vs. those receiving vehicle by day 7 for scale and erythema, and by day 14 for thickness. No significant improvement, however, was seen on day 3 . None of the immunohistological markers (CD1a, CD4, CD8, ICAM-1, VCAM-1, E-selectin, HLA-DR) semiquantitatively assessed in psoriatic plaques was significantly changed by calcipotriene treatment for 7 days. In the calcipotriene-treated group, interleukin (IL)-10 levels ( $\mathrm{pg} / \mu \mathrm{g}$ of protein) increased by $57 \%$ from baseline $(0 \cdot 030 \pm 0 \cdot 006$; mean \pm SEM) to day $3(0 \cdot 047 \pm 0 \cdot 011)(P=0.05$ vs. baseline; $n=10)$ and remained elevated at day $7(0 \cdot 046 \pm 0 \cdot 012)$. IL-8 levels $(\mathrm{pg} / \mu \mathrm{g}$ of protein), however, declined by $70 \%$ from baseline $(0 \cdot 13 \pm 0 \cdot 06)$ to day $3(0 \cdot 04 \pm 0 \cdot 01)$, and remained low at day $7(0 \cdot 03 \pm 0 \cdot 02)(P<0 \cdot 05$ vs. baseline; $n=10)$. Both IL- 8 and IL-10 were unaffected by vehicle treatment. Calcipotriene-induced clinical improvement of psoriasis is preceded by an increase in IL10 and a concomitant decrease in IL-8 levels. The changes in the level of these two cytokines provide further evidence for immunological changes as a significant part of the mechanism of action of calcipotriene in psoriasis.

Calcipotriene, also known as calcipotriol and MC-903, is a synthetic vitamin $\mathrm{D}_{3}$ analogue established to be effective topically in the treatment of psoriasis. ${ }^{1-3}$ The proposed mechanisms by which calcipotriene improves psoriasis have centred around keratinocytes. The ability of calcipotriene and 1,25-dihydroxyvitamin $\mathrm{D}_{3}$ $\left(1,25(\mathrm{OH})_{2} \mathrm{D}_{3}\right)$ to inhibit keratinocyte proliferation and to induce differentiation is compatible with their antipsoriatic actions. ${ }^{4-6}$ However, whether these observations made primarily in vitro are also operational in vivo require further studies. Furthermore, as many

Correspondence: Sewon Kang. E-mail: swkang@umich.edu This work was presented in part at Psoriasis: from Gene to Clinic, London, U.K., December 1996, and Society for Investigative Dermatology, Washington, DC, U.S.A., April 1997. abnormal parameters measurable in lesional skin of psoriasis normalize as the lesion improves clinically, an appropriate temporal relationship must be demonstrated in vivo, before any claim of cause and effect can be made. Although psoriasis is a disease characterized by keratinocyte hyperproliferation and incomplete differentiation, mounting scientific evidence points to an immune-mediated disorder, possibly an organ-specific autoimmune disease of skin. ${ }^{7-9}$ Indeed, many effective therapeutic agents for psoriasis are immunosuppressive in nature. Emerging evidence indicates that $1,25(\mathrm{OH})_{2} \mathrm{D}_{3}$ possesses immunomodulatory activities. In several animal models of autoimmune diseases, $1,25(\mathrm{OH})_{2} \mathrm{D}_{3}$ and its analogues have been shown to prevent the onset or retard the progression of such 
diseases. ${ }^{10-12}$ In this report, we provide evidence that very early in the treatment (within a few days), and several days before clinical improvement is detectable, calcipotriene modulates two important cytokines in a direction that may lead to the resolution of psoriasis.

\section{Patients and methods}

\section{Patients and design}

Thirty patients with mild to moderate, stable, plaquetype psoriasis (20 men, 10 women; mean age 41 ; age range $18-66$ years) were entered into this double-blind, placebo-controlled study. All patients had been without systemic therapy, including ultraviolet (UV) radiation, for psoriasis for 4 weeks, and topical therapy, other than emollients, for 2 weeks. During the study, natural or artificial sunlight exposure was discouraged. None of the women was pregnant or breast-feeding. The study was approved by the institutional review board of the University of Michigan Medical Center, and all subjects gave written, informed consent.

\section{Treatment}

A computer-generated code was used to randomly assign 15 subjects to calcipotriene treatment (11 men and four women) and 15 subjects to treatment with vehicle (nine men and six women). The calcipotriene 0.005\% ointment (Bristol-Myers Squibb Corp., Buffalo, NY, U.S.A.) or colour-matched vehicle were in identical tubes, so that neither the investigators nor the patients knew the treatment group to which any patient was assigned. The study medication was applied by the patients twice daily for 6 weeks to their psoriatic plaques. Patients were instructed not to apply the study medication within $8 \mathrm{~h}$ of evaluation.

\section{Clinical evaluation}

Clinical evaluation of all patients was performed by two investigators (L.F. and S.K.) at baseline (day 0), after 3, 7 and 14 days of treatment, and then biweekly for a total of 6 weeks. For any given patient, only one investigator performed the clinical evaluation at all the visits. At each visit, the overall erythema, thickness and scaling of psoriatic plaques were graded on a scale of 0 to 8 where 0 is none and 8 is the most severe. More specifically, for erythema ( 0 , no evidence of erythema; 2 , lightly red; 4 , red; 6 , very red; 8 , extremely red), for thickness $(0$, no evidence of plaque above normal skin level; 2 , slight but definite elevation above normal skin level; 4, moderate elevation with rounded or sloped edges to plaque; 6 , marked elevation with sharp edges to plaque; 8 , very marked elevation with very hard sharp edges to plaque), and for scale ( 0 , no evidence of scaling on the lesions; 2 , mild: mainly fine scales, some lesions at least partially covered; 4, moderate: somewhat coarser scales, most lesions at least partially covered; 6 , severe: thick scales, virtually all lesions covered; 8 , very severe: very thick scales, all lesions covered). In addition, global response to treatment was graded independently at the end of therapy (week 6) on a scale of -1 to $5(-1$, worse than at baseline evaluation; 0 , no change from baseline; 1 , slight improvement (about 25\%), however, significant evidence of psoriasis remains; 2 , moderate improvement (about 50\%), intermediate between marked and slight improvement; 3, marked improvement (about 75\%), some evidence of psoriasis remains; 4, almost clear, very significant clearance with only minor evidence of psoriasis; 5, completely clear with no evidence of psoriasis).

\section{Laboratory assessment}

In addition to the clinical assessment, the following laboratory tests were performed at baseline and weeks 2 and 6: full blood count, serum biochemistry screen including calcium and phosphorus, and routine urinalysis.

\section{Immunohistological analysis}

Before, and at days 3 and 7 of therapy, a 6-mm diameter punch biopsy specimen was taken from a target psoriatic plaque on the trunk or proximal extremities. The biopsy sites were at the periphery of plaques, $1 \mathrm{~cm}$ in from the edges and separated from each other by at least $2 \mathrm{~cm}$. Lidocaine (1\%) without adrenaline was used as the local anaesthetic. These specimens were embedded in OCT medium (Miles Laboratories, Naperville, IL, U.S.A.), frozen in liquid nitrogen, and stored at $-70^{\circ} \mathrm{C}$ until use. Five-micrometre sections were cut on a cryostat and stained with a panel of monoclonal antibodies, namely Leu 6 (anti-CD1a: Becton Dickinson, Burlingame, CA, U.S.A.), T4 (anti-CD4: Coulter, Hialeah, FL, U.S.A.), T8 (anti-CD8: Coulter), anti-ICAM-1 (R\&D Systems, Minneapolis, MN, U.S.A.), anti-VCAM-1 (R\&D Systems), anti-E-selectin (R\&D Systems), and L243 (anti-HLA-DR: Becton-Dickinson). A standard immunoperoxidase technique (Vectastain $\mathrm{ABC}$ Kit, Vector Laboratories, Burlingame, CA, U.S.A.) was used with 
3-amino-9-ethyl carbazole as the chromogen and 1\% haematoxylin as the counterstain. All immunohistological sections were examined by one of us (J.H.C.) who was not involved in the clinical part of the study. Each patient's specimens were randomized and blinded. The staining intensity of each marker was graded under light microscopy using a 0 to 5 six-point scale in which 0 indicated no staining; 1 , minimal staining; 2 , low-tomoderate levels of staining; 3, moderate staining; 4, high levels of staining; and 5, maximal staining.

\section{Enzyme-linked immunosorbent assay (ELISA) of cytokines}

A second set of 6-mm punch biopsies was obtained from the target psoriatic plaque at baseline and days 3 and 7 of therapy from all subjects. The biopsy sites were again at the periphery of psoriatic plaques, $1 \mathrm{~cm}$ in from the edges. If the target lesion were not large enough to accommodate six 6-mm punch biopsies, each at least $2 \mathrm{~cm}$ apart, a similar lesion in close proximity to the target plaque was selected for ELISA analysis. Specimens were snap-frozen in liquid nitrogen and stored at $-70{ }^{\circ} \mathrm{C}$ until use. The frozen skin specimens were homogenized using a $2 \mathrm{~mL}$ glass homogenizer on ice in a $1.2 \mathrm{~mL}$ solution of $20 \mathrm{mmol} / \mathrm{L}$ Tris- $\mathrm{HCl} \mathrm{pH} 7 \cdot 4$, $1 \mathrm{mmol} / \mathrm{L}$ phenylmethylsulphonyl fluoride and $1 \mu \mathrm{g} / \mathrm{mL}$ of each of apropitin and leupeptin, as previously described. $^{13}$ The homogenates were then centrifuged at $11,750 \mathrm{~g}$ at $4^{\circ} \mathrm{C}$ for $5 \mathrm{~min}$. The supernatants were assayed by the following ELISA kits: interleukin (IL)-2, IL-10 and interferon- $\gamma$ (IFN- $\gamma$ ) (Genzyme Co., Cambridge, MA, U.S.A.) and IL-8 (T Cell Diagnostics, Inc., Woburn, MA, U.S.A.).

\section{Statistical analysis}

Changes in clinical and immunohistological variables of calcipotriene- and vehicle-treated patients were statistically compared using Student's two sample $t$-test. Timerelated, within group comparisons were made with the paired $t$-test or the repeated measures analysis of variance for multiple time-points. Linear regression techniques were used to determine the concentration of cytokines (ELISA variables) from the derived curves based on a test of known standards. Time-related differences were then assessed with the paired $t$-test. The clinical global response was compared between treatment groups using the $\chi^{2}$ test. Summary statistics are expressed as mean \pm SEM. All P-values are twotailed. The data were analysed with MIDAS (Michigan Interactive Data Analysis System) and SAS ${ }^{\circledR}$ (SAS Institute Inc., Cary, NC, U.S.A.).

\section{Results}

\section{Clinical parameters}

Thirty patients, 15 in each treatment group, were enrolled and all completed the study. Before treatment, the severity of psoriasis in the calcipotriene and placebo groups was comparable $(P>0 \cdot 3)$ (Fig. 1). After 6 weeks of therapy, the reductions in scale, erythema and thickness were significantly greater in the calcipotriene group as compared with the placebo group $(P<0 \cdot 05)$. At the end of treatment, psoriasis in the calcipotriene
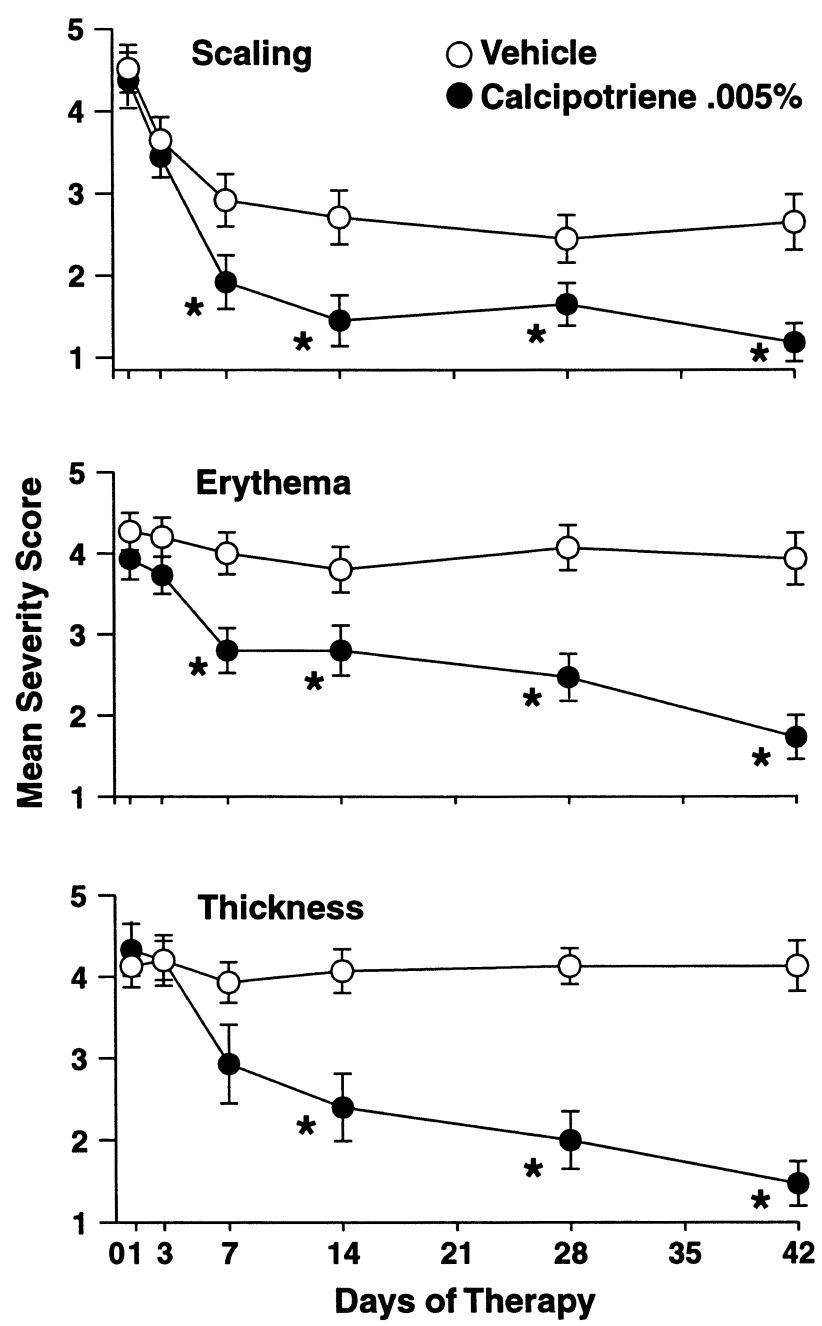

Figure 1. Overall change in psoriasis severity (scale, erythema, thickness) based on a scale of $0-8$ (see text), during treatment with calcipotriene and vehicle. Values are mean \pm SEM. Statistically significant and clinically appreciable improvements were first attained after 7 days for scale and erythema, and after 14 days for thickness, with calcipotriene and were sustained for the remainder of the study. Asterisks $\left(^{*}\right)$ indicate a significant difference $(P<0 \cdot 05)$ between groups. 
group showed significant improvement in the global response from baseline compared with the vehicle group $(P<0.05)$; of the 15 patients who received $0.005 \%$ calcipotriene, psoriasis was completely clear in three $(20 \%)$, markedly improved to almost clear in five (33\%), moderately improved in five (33\%) and slightly improved in two (13\%) (Fig. 2). Of the 15 patients who received vehicle, none showed marked or better improvement, six $(40 \%)$ showed slight to moderate improvement, and the remaining nine (60\%) demonstrated no change or worsening (Fig. 2). Statistically significant $(P<0.05)$ improvement in scale and erythema of psoriasis in the calcipotriene group, as compared with the vehicle group, was attained after 7 days of treatment. For thickness of psoriatic lesions, statistically significant improvement in the calcipotriene group, as compared with the vehicle, did not occur until week 2 of therapy. All of the clinical parameters assessed for target psoriatic plaques improved progressively with calcipotriene treatment.

\section{Side-effects and laboratory changes}

All 30 patients who enrolled in the study completed the entire 6 weeks of therapy without serious adverse events. Two of the 15 subjects who received calcipotriene experienced mild irritation with redness and scaling on the face and clinically normal skin surrounding psoriatic plaques. Clinical laboratory parameters, including the serum calcium and phosphorus levels, did not change significantly during or after 6 weeks of calcipotriene therapy.

\section{Immunohistological study}

During the first week of therapy, there were no significant differences between calcipotriene and vehicle groups for any of the immunohistological markers examined.

\section{ELISA analysis}

Biopsy specimens from the first 10 patients (subject numbers 1-10; five in the calcipotriene and five in the vehicle groups) were processed according to an ELISA protocol recommended by a pathology laboratory. This procedure, however, was inappropriate for skin tissue samples as all the extracts were over-diluted. Attempts to reconcentrate the extracts were not successful. A modified protocol for ELISA was used for the remaining biopsy specimens (subject numbers 11-30; 10 in the calcipotriene and 10 in the vehicle groups). Therefore, data are presented for 20 patients. With calcipotriene therapy, the IL-10 levels (pg/ $\mu \mathrm{g}$ of protein) increased by $57 \%$ from baseline $(0 \cdot 030 \pm 0 \cdot 006)$ to day 3 $(0 \cdot 047 \pm 0 \cdot 011)(P=0.05$ vs. baseline; $n=10)$ and remained elevated $(0 \cdot 046 \pm 0 \cdot 012)$ at day 7 (Fig. 3a). The IL- 8 levels ( $\mathrm{pg} / \mu \mathrm{g}$ of protein) decreased by $70 \%$ in lesional skin treated with calcipotriene from $0 \cdot 13 \pm 0.06$ at baseline to $0.04 \pm 0.01$ at day 3 , and remained low $(0.03 \pm 0.02)$ at day $7(P<0.05$ vs. baseline; $n=10$ ) (Fig. 3b). Neither IL-8 nor IL-10 levels were affected by vehicle treatment. In our ELISA-based system, IL-2 and IFN- $\gamma$ were not detectable.

\section{Discussion}

Consistent with previous reports, ${ }^{1,3,14}$ we found calcipotriene ointment to be significantly more effective than its vehicle in improving psoriasis. Unlike almost all earlier studies with topical calcipotriene in which the first follow-up evaluation was performed after 7 or 14 days of therapy, we included an examination of our patients at day 3. At this early time-point, calcipotriene had produced no statistically significant or clinically perceivable improvement in psoriasis. The first demonstration of statistically significant improvement in clinical parameters occurred after 7 days of therapy with

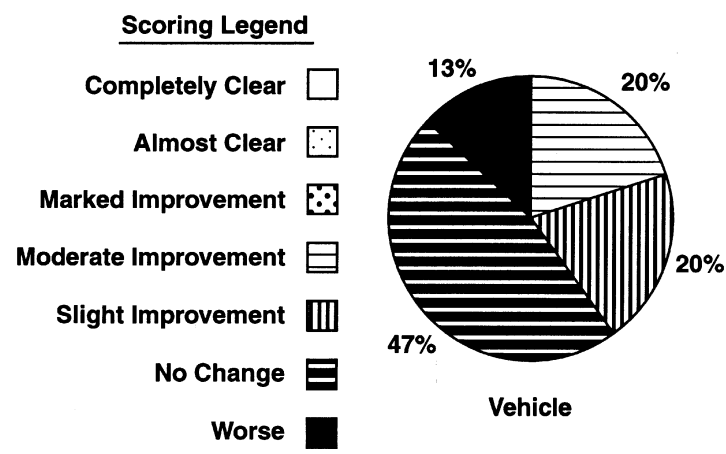

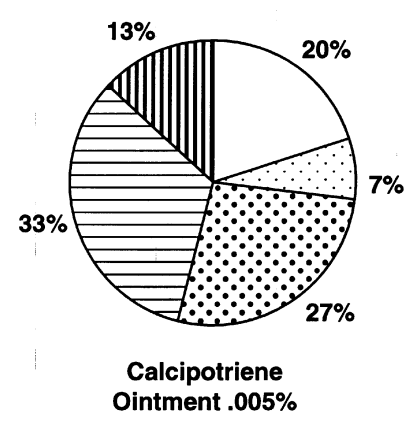

Figure 2. Overall global response after 6 weeks of treatment with calcipotriene or vehicle ointment for psoriasis. As compared with vehicle, calcipotriene produced significant improvement of psoriasis $(P<0 \cdot 01)$. Fifteen patients received calcipotriene and 15 patients received vehicle ointment. 


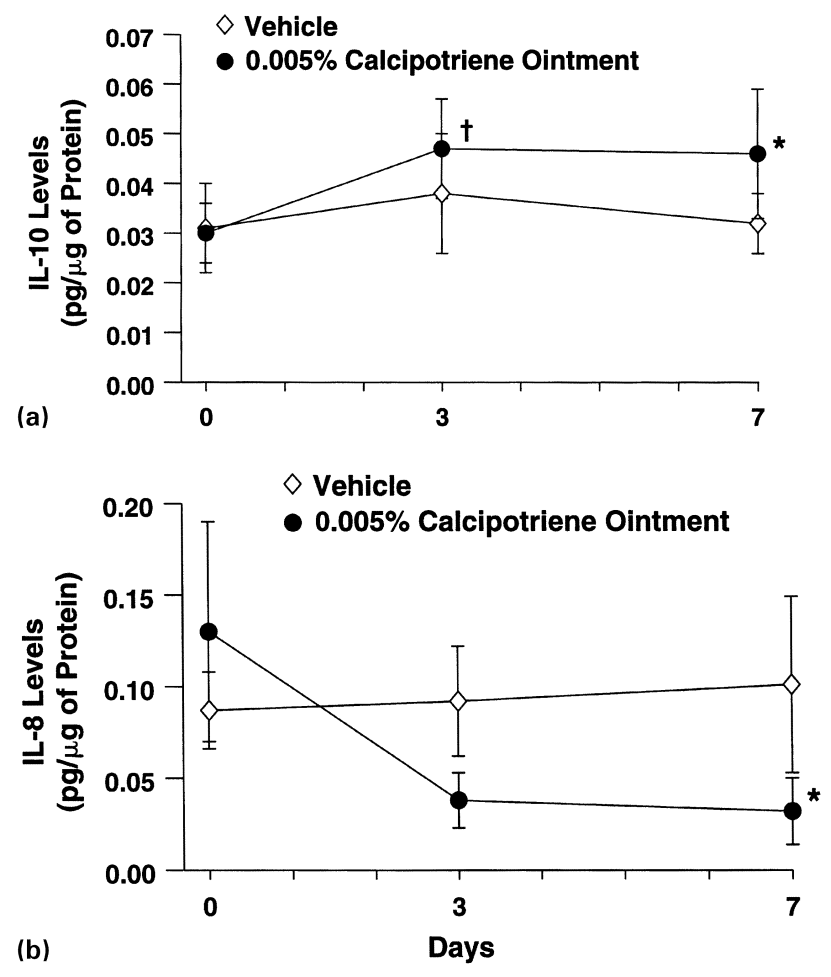

Figure 3. Mean levels of IL-10 (a) and IL-8 (b) in the lesional skin of psoriasis during treatment with calcipotriene $(n=10)$ and its vehicle $(n=10)$. ELISA conditions and analytical procedures are described in Materials and methods. Values are mean \pm SEM. Asterisk $(*)$ indicates $P<0.05$ and dagger $(\dagger)$ indicates $P=0.05$ vs. baseline.

calcipotriene for scale and erythema. For the thickness, significant improvement was first noted after 2 weeks of calcipotriene therapy. This time-line in the improvement of clinical parameters observed in our patients was similar to that in previous studies. ${ }^{1,3,14}$

Immunohistochemical assessment of psoriatic skin during the first week of therapy revealed that in both the epidermal and dermal compartments, the infiltration of T-helper $(\mathrm{CD} 4+)$ or T-suppressor $(\mathrm{CD} 8+)$ cells was not significantly changed. This is consistent with previous observations that even in psoriatic plaques that show clinical response to topical calcipotriene, when examined histologically, the mononuclear infiltrate remains largely unchanged. ${ }^{15,16}$ More recently, Lu et al. ${ }^{17}$ reported that psoriatic plaques biopsied when they showed clearing of at least the centre or after 2 months of treatment with topical $1,25(\mathrm{OH})_{2} \mathrm{D}_{3}$ $(0 \cdot 005 \%)$, still demonstrated considerable numbers of intraepidermal T cells, as compared with vehicle. Similarly, cells expressing HLA-DR, CD1a, or adhesion molecules (ICAM-1, VCAM-1 and E-selectin) remained unchanged in staining intensity by calcipotriene therapy during the first 7 days.
Despite the lack of clinical and immunohistochemical changes early in the treatment, IL-10 levels increased in the lesional skin of psoriasis after 3 days of calcipotriene therapy. IL-10 is a type 2 (T2) cytokine which has been shown to inhibit antigen-specific activation and proliferation of $\mathrm{T}$ cells. ${ }^{18,19}$ These inhibitory effects are mediated through antigen-presenting cell function, ${ }^{20,21}$ including the suppression of expression of B7-1 (CD80) and B7-2 (CD86) which are costimulatory molecules necessary for T-cell activation. ${ }^{22,23}$ IL-10 has also been shown directly to suppress IL-2 secretion by CD4+ T cells. $^{24,25}$ Based on these and other properties, IL-10 has been referred to as an immunosuppressive or antiinflammatory cytokine. Indeed, a recent demonstration that immunosuppressive response to UV radiation was impaired in IL-10-deficient, gene knock-out mice, further strengthens the immunosuppressive role of this cytokine. ${ }^{26}$ In UV-induced IL-10 production, DNA damage in keratinocytes and perhaps other skin cells appears to be the initiating signal. ${ }^{27}$ Although the mechanism by which calcipotriene induces $\mathrm{IL}-10$ is unknown, it is highly unlikely to be through a similar pathway. Therefore, the level of IL-10 produced in psoriatic skin following a combination of UV-irradiation and calcipotriene treatment is likely to be greater than by each agent alone. This may partly explain the enhanced clinical response of psoriasis to the combination therapy. ${ }^{28,29}$

The ability of calcipotriene to induce IL-10 is also relevant in the context of our current understanding of T-cell-mediated immune responses. Initially described in mice, ${ }^{30}$ then subsequently demonstrated in humans, ${ }^{31,32}$ immune responses mediated by lymphocytes can be classified into fairly discrete and mutually exclusive reactions termed type 1 (T1) or T2. T1 represents a strong cellular immune response and is characterized by elaboration of cytokines IL- 2 , IFN- $\gamma$ and IL-12. On the other hand, T2 represents an enhanced humoral response associated with secretion of IL-4, IL-5 and IL-10. Not only are T1 and T2 responses fairly discrete, they are self-reinforcing in that $\mathrm{T} 1$ cytokines tend to inhibit $\mathrm{T} 2$ cytokine production, and vice versa. ${ }^{33}$ In psoriasis, the predominant cytokines expressed by lesional T cells belong to T1 (IL-2 and IFN- $\gamma){ }^{7-9}$ Therefore, IL-10 production induced by calcipotriene would antagonize the $\mathrm{T} 1$ state, and shift the immune response from $\mathrm{T} 1$ towards $\mathrm{T} 2$, which would have a therapeutic effect in psoriasis. By the same token, however, IL-10 induction is expected not to have a beneficial effect in a disease characterized by $\mathrm{T} 2$ cytokine expression (IL-4, IL-5, IL-10) such as atopic 
dermatitis. $^{31}$ In our ELISA-based system, we were unable to measure elevated levels of IL- 2 and IFN- $\gamma$ in untreated psoriatic plaques. For these two cytokines, the use of skin extracts prepared from the whole punch biopsy specimens may have been too crude and dilute for proper detection.

Concomitant with an early increase in IL-10, IL-8 levels were reduced in plaques of psoriasis treated with calcipotriene. This is consistent with in vitro data in which $1,25(\mathrm{OH})_{2} \mathrm{D}_{3}$ has been shown to inhibit IL-1induced IL-8 mRNA and protein in normal human keratinocytes, fibroblasts and peripheral blood mononuclear cells. $^{34}$ IL- 8 is a proinflammatory chemokine that is chemotactic for polymorphonuclear cells ${ }^{35}$ as well as $\mathrm{T}$ lymphocytes. ${ }^{36}$ Furthermore, it promotes proliferation of keratinocytes. ${ }^{37}$ More recently, IL-8 has been shown to be a potent angiogenic factor, ${ }^{38,39}$ as well as an inducer of nitric oxide synthase. ${ }^{40}$ These multiple properties, and the fact that IL- 8 and its receptor are markedly increased in psoriatic epidermis, ${ }^{41,42}$ indicate that IL- 8 may be an important mediator of the psoriatic process. Therefore, a reduction of this inflammatory cytokine by calcipotriene could be involved in the therapeutic action of calcipotriene in psoriasis.

Our immunohistological data indicated that after 7 days of therapy with calcipotriene, there were no detectable changes in the expression of cell markers and adhesion molecules. The clear and early alterations in levels of IL-10 (an increase) and IL-8 (a decrease) suggest that although the cells identifiable with various immune markers remained in the involved plaques of psoriasis, there were significant functional changes induced by the drug. Based on our findings and those of others, ${ }^{15-17}$ there is clearly a lag-time that is longer than a week of therapy, between the cytokine modulations and a significant reduction in expression of these immune markers. An important factor in this regard probably involves the turnover rate of some of these markers.

We did not study the cell type(s) responsible for the elaboration of the cytokines in this study. At least in CD4+ T lymphocytes, IL-8 induces its own production, and both spontaneous as well as cytokine-induced IL-8 production is inhibited by IL-10. ${ }^{43}$ It is possible then, that the reduction in IL- 8 may be secondary to the induction of IL-10 by calcipotriene therapy. Regardless of the cellular source, and the possible inter-relationship in the expression of the two cytokines, the fact that IL10 induction and IL-8 reduction occurred early in the treatment, prior to the observation of significant clinical or immunohistological improvement, indicates that these cytokine alterations are not simply secondary to the process of resolution of a psoriatic plaque. Rather, the time-line is compatible with the possibility that elaboration of the immunosuppressive cytokine (IL-10) and a concomitant reduction in the proinflammatory cytokine (IL-8) may mediate at least part of the immunopharmacological improvement in psoriasis. Therefore, in addition to keratinocyte hyperproliferation and incomplete differentiation, the local cytokine imbalance observed in psoriatic lesions appears to be corrected at least in part by calcipotriene.

\section{Acknowledgments}

This study was supported in part by a grant from the Bristol-Myers Squibb Corp., Buffalo, NY, U.S.A., which had no part in the analysis, interpretation or reporting of the results, and by the Babcock Dermatologic Endowment of the Department of Dermatology, University of Michigan, Ann Arbor, MI, U.S.A. Dr Kang is a recipient of a Dermatology Foundation Clinical Career Development Award. We thank Dr John J.Voorhees for his advice and critical review of this manuscript.

\section{References}

1 Kragballe K. Treatment of psoriasis by the topical application of the novel cholecalciferol analogue calcipotriol (MC 903). Arch Dermatol 1989; 125: 1647-52.

2 Cunliffe WJ, Berth-Jones J, Claudy A et al. Comparative study of calcipotriol (MC 903) ointment and betamethasone 17-valerate ointment in patients with psoriasis vulgaris. J Am Acad Dermatol 1992; 26: 736-43.

3 Kragballe K, Beck HI, Sogaard H. Improvement of psoriasis by a topical vitamin $\mathrm{D}_{3}$ analogue (MC903) in a double-blind study. $\mathrm{Br} \mathrm{J}$ Dermatol 1988; 199: 223-30.

4 Smith EL, Walworth NC, Holick MF. Effect of 1,25-dihydroxyvitamin $\mathrm{D}_{3}$ on the morphological and biochemical differentiation of cultured human epidermal keratinocytes grown in serum-free conditions. J Invest Dermatol 1986; 86: 709-14.

5 Kragballe K, Wildfang IL. Calcipotriol (MC903), a novel noncalcipotropic vitamin $\mathrm{D}_{3}$ analogue, stimulates terminal differentiation and inhibits proliferation of cultured human keratinocytes. Arch Dermatol Res 1990; 282: 164-7.

6 Bikle DD, Gee E, Pillai S. Regulation of keratinocyte growth, differentiation, and vitamin D metabolism by analogs of 1,25dihydroxyvitamin D. J Invest Dermatol 1993; 101: 713-18.

7 Barker JNWN. The immunopathology of psoriasis. Baillière's Clin Rheumatol 1994; 429-37.

8 Griffiths CEM, Voorhees JJ. Immunological mechanisms involved in psoriasis. Springer Semin Immunopathol 1992; 13: 441-54.

9 Christophers E. The immunopathology of psoriasis. Int Arch Allergy Immunol 1996; 110: 199-206.

10 Binderup L. Immunological properties of vitamin D analogues and metabolites. Biochem Pharmacol 1992; 43: 1885-92. 
11 Lemire JM. Immunomodulatory actions of 1,25-dihydroxyvitamin D. J Steroid Biochem Mol Biol 1995; 53: 599-602.

12 Cantorna MT, Hayes CE, DeLuca HF. 1,25-Dihydroxyvitamin $\mathrm{D}_{3}$ reversibly blocks the progression of relapsing encephalomyelitis, a model of multiple sclerosis. Proc Natl Acad Sci USA 1996; 93: 7861-4.

13 Nickoloff BJ, Kunkel SL, Burdick M, Strieter RM. Severe combined immunodeficiency mouse and human psoriatic skin chimeras: validation of a new animal model. Am J Pathol 1995; 146: 580-8.

14 Dubertret L, Wallch D, Souteyrand P et al. Efficacy and safety of calcipotriol (MC 903) ointment in psoriasis vulgaris. J Am Acad Dermatol 1992; 27: 983-8.

15 DeJong EMGJ, van de Kerkhof PCM. Simultaneous assessment of inflammation and epidermal proliferation in psoriatic plaques during long-term treatment with the vitamin $\mathrm{D}_{3}$ analogue MC903: modulations and interrelations. Br J Dermatol 1991; 124: 221-9.

16 Verburgh CA, Nieboer C. Local application of vitamin $\mathrm{D}_{3}$ derivative MC903 in psoriasis: influence on cellular infiltrate, Langerhans cells and keratinocyte (KC) markers. J Invest Dermatol 1989; 93: 310 (Abstr.).

17 Lu I, Gilleaudeau P, McLane JA etal. Modulation of epidermal differentiation, tissue inflammation, and T-lymphocyte infiltration in psoriatic plaques by topical calcipotriol. J Cutan Pathol 1996; 23: 419-30.

18 Yssel H, de Waal Malefyt R, Roncarolo MG et al. IL-10 is produced by subsets of human $\mathrm{CD} 4^{+}$cell clones and peripheral blood T cells. J Immunol 1992; 149: 2378-84.

19 de Waal Malefyt R, Haanen J, Spits H et al. Interleukin 10 (IL-10) and viral IL-10 strongly reduce antigen-specific human T cell proliferation by diminishing the antigen-presenting capacity of monocytes via downregulation of class II major histocompatibility complex expression. J Exp Med 1991; 174: 915-24.

20 Caux C, Massacrier C, Vanbervliet B et al. Interleukin inhibits T cell alloreaction induced by human dendritic cells. Int Immunol 1994; 6: $1177-85$.

21 Ding L, Shevach EM. IL-10 inhibits mitogen induced T cell proliferation by selectively inhibiting macrophage costimulatory function. J Immunol 1992; 148: 3133-9.

22 Ding L, Linsley PS, Huang LY etal. IL-10 inhibits macrophage costimulatory activity by selectively inhibiting the up-regulation of B7 expression. J Immunol 1993; 151: 1224-34.

23 Chang CH, Furue M, Tamaki K. B7-1 expression of Langerhans cells is up-regulated by proinflammatory cytokines, and is downregulated by interferon-gamma or by interleukin-10. Eur J Immunol 1995; 25: 394-8.

24 Taga K, Mostowski K, Tosato G. Human interleukin-10 can directly inhibit T-cell growth. Blood 1993; 81: 2964-71.

25 de Waal Malefyt R, Yssel H, de Vries JE. Direct effects of IL-10 on subsets of human $\mathrm{CD}^{+}{ }^{+} \mathrm{T}$ cell clones and resting T cells. Specific inhibition of IL-2 production and proliferation. J Immunol 1993; 150: 4754-65.

26 Beissert S, Hosoi J, Kühn R etal. Impaired immunosuppressive response to ultraviolet radiation in interleukin-10-deficient mice. $J$ Invest Dermatol 1996; 107: 553-7.

27 Nishigori C, Yarosh DB, Ullrich SE et al. Evidence that DNA damage triggers interleukin 10 cytokine production in UV-irradiated murine keratinocytes. Proc Natl Acad Sci USA 1996; 93: 10354-9.

28 Kragballe K. Combination of topical calcipotriol (MC 903) and UVB radiation for psoriasis vulgaris. Dermatologica 1990; 181: 211-14.

29 Kokelj F, Lavaroni G, Guadagnini A. UVB versus UVB plus calcipotriol (MC 903) therapy for psoriasis vulgaris. Acta Derm Venereol (Stockh) 1995; 75: 386-7.

30 Mosmann TR, Cherwinski K, Bond MW et al. Two types of murine helper T-cell clones. 1. Definition according to profiles of lymphokine activities and secreted proteins. J Immunol 1986; 136: $2348-$ 57.

31 Wierenga EA, Snoek M, de Groot C et al. Evidence for compartmentalization of functional subsets of $\mathrm{CD}^{+} \mathrm{T}$ lymphocytes in atopic patients. J Immunol 1990; 144: 4651-6.

32 Parronchi P, Macchia D, Piccinni MP et al. Allergen- and bacterial antigen-specific T-cell clones established from atopic donors show different profile of cytokine production. Proc Natl Acad Sci USA 1991; 88: 4538-42.

33 Del Prete G, Maggi E, Romagnani S. Biology of disease. Human Thl and Th2 cells: functional properties, mechanisms of regulation, and role in disease. Lab Invest 1994; 70: 299-305.

34 Larsen CG, Kristensen M, Paludan K et al. $1,25(\mathrm{OH})_{2}-\mathrm{D}_{3}$ is a potent regulator of interleukin-1 induced interleukin-8 expression and production. Biochem Biophys Res Commun 1991; 176: 10206.

35 Matsushima K, Oppenheim JJ. Interleukin 8 and MCAF: novel inflammatory cytokines inducible by IL-1 and TNF. Cytokines 1989; $1: 2-13$.

36 Larsen CG, Anderson AO, Apella E et al. The neutrophil-activating protein (NAP-1) is also chemotactic for T lymphocytes. Science 1989; 243: 1464-6.

37 Tuschil A, Lam C, Halsberger A, Lindley I. Interleukin-8 stimulates calcium transients and promotes epidermal cell proliferation. $J$ Invest Dermatol 1992; 99: 294-8.

38 Koch AE, Polverini PJ, Kunkel SL et al. Interleukin-8 as a macrophage-derived mediator of angiogenesis. Science 1992; 258: $1798-801$.

39 Nickoloff BJ, Mitra RS, Varani J etal. Aberrant production of interleukin- 8 and thrombospondin-1 by psoriatic keratinocytes mediates angiogenesis. Am J Pathol 1994; 144: 820-8.

40 Bruch-Gerharz D, Fehsel K. Suschek C et al. A proinflammatory activity of interleukin 8 in human skin: expression of the inducible nitric oxide synthase in psoriatic lesions and cultured keratinocytes. J Exp Med 1996; 184: 2007-12.

41 Gillitzer R, Berger R, Mielke V et al. Upper keratinocytes of psoriatic skin lesions express high levels of NAP-1/IL-8 mRNA in situ. $J$ Invest Dermatol 1991; 97: 73-9.

42 Sticherling M, Bornscheuer E, Schroder J-M, Christophers E. Localization of neutrophil-activating peptide-1/interleukin-8immunoreactivity in normal and psoriatic skin. J Invest Dermatol 1991; 96: 26-30.

43 Gesser B, Deleuran B, Lund M et al. Interleukin-8 induces its own production in $\mathrm{CD} 4^{+} \mathrm{T}$ lymphocytes: a process regulated by interleukin 10. Biochem Biophys Res Commun 1995; 210: 660-9. 